\title{
A Video-based Hyper-focal Imaging Method For Iris Recognition In The Visible Spectrum
}

\author{
Sriram Pavan Tankasala, Vikas Gottemukkula, \\ Sashi Kanth Saripalle, Venkata Goutam Nalamati, \\ Reza Derakhshani \\ Dept. of Computer Science and Electrical Engineering, \\ University of Missouri - Kansas City
}

\author{
Raghunandan Pasula, Arun Ross \\ Lane Department of Computer Science and \\ Electrical Engineering, \\ West Virginia University
}

\begin{abstract}
We design and implement a hyper-focal imaging system for acquiring iris images in the visible spectrum. The proposed system uses a DSLR Canon T2i camera and an Okii controller to capture videos of the ocular region at multiple focal lengths. The ensuing frames are fused in order to yield a single image with higher fidelity. Further, the proposed setup extends the imaging depth-of-field (DOF), thereby preempting the need for employing expensive cameras for increased DOF. Experiments convey the benefits of utilizing a hyper-focal system over a traditional fixed-focus system for performing iris recognition in visible spectrum.
\end{abstract}

Keywords: hyper-focal, depth of field, iris recognition and focus

\section{INTRODUCTION}

The texture of the iris is typically imaged in the near infrared (NIR) spectrum. This is because the texture of dark-colored irides is more easily discernible in the NIR spectrum rather than the visible (RGB) spectrum. However, recent work has explored the possibility of conducting iris recognition in the visible spectrum using RGB images [3], [7], [14], [15], [16]. Iris recognition using RGB images is challenging for darker irides due to the effect of melanin. Darker irides have a higher concentration of melanin that absorbs most of the visible light and the images appear dark due to the low power of the reflected light. The effect of melanin decreases significantly in the NIR spectrum thereby revealing textural patterns even for darker irides [4]. Iridal information in visible spectrum can be improved by using a controlled lighting setup to illuminate the structure of the iris and producing sufficient reflected light towards the imaging camera. However, due to the required higher intensity illumination for visible light iris captures, especially for darker eyes, the placement of such illuminators should be carefully studied in order to avoid user discomfort.

Quality of an acquired iris image is a very important metric that directly affects the performance of an iris recognition system. Image focus plays a predominant role in estimating image quality [2], [6]. Better focused images can be only acquired at the time of image acquisition and cannot be easily compensated for once the process is complete ${ }^{1}$. A higher focus value is preferred for better quality images. Camera's depth of field (DOF) is defined as physical depth of the volume being imaged that appears in-focus on the capture

\footnotetext{
${ }^{1}$ Exceptions include Lytro Camera (https://www.lytro.com/camera\#) and Throwable Panoramic Ball Camera (http://jonaspfeil.de/ballcamera)
}

image. Another important factor in iris image quality is shutter speed, as shorter exposures result in less motion blur. Yet the corresponding reduced exposure times require larger camera apertures that, in turn, reduce DOF and cause the image to be partially out of focus. Most importantly, the illumination level has to be high for visible spectrum iris image acquisition in order to better expose texture of darker irides, which in turn requires either longer exposure times (leading to motion blur) or larger apertures which lead to shallower DOFs, or both. As mentioned earlier, simply increasing the intensity of illuminator may result in subject discomfort and is not practical. The aforementioned opposing requirements create further challenges for visible light iris recognition.

In this paper, we propose an imaging method using a combination of focus bracketing with lateral white LED lighting as a solution to overcome the aforementioned RGB iris exposure and depth of field problems. A traditional approach to increase the DOF is to increase the f-number of the lens. However, as mentioned earlier, increasing the f-number decreases the optics aperture and requires longer exposure time or higher illumination intensity. To better expose irides, especially those with heavy pigmentation, we allow for larger apertures. As a result, a single close up frame of the ocular area will be only partially in-focus. Next, multiple captures of the same ocular area are taken in rapid succession but each at a slightly different focal plane (focus bracketing technique), and the stack of images is then fused to improve overall focus. Focus bracketing, also known as hyper-focal imaging, is a technique to obtain a single sharp, high DOF image from a series of images captured at varying focal distances. In this paper, we demonstrate the benefits of this technique for RGB ocular biometrics.

Earlier work on overcoming the focus problem [13], [11], [17] use wavefront coding to achieve extended DOF at a fixed focus. In [12], the authors use specular reflection of the IRLED illuminator to rapidly assess the focus of a sequence of frames acquired at varying focal lengths and select the best focused frames for iris recognition. In [5], the authors use auto-focus feature based on lens design and self-alignment to position the iris in the camera's focal volume. The system in [4] maximizes the spectral power in the middle and upper frequencies of the 2D Fourier spectrum of the acquired images in order to select the best frame for iris recognition. 
In this paper we introduce a novel iris imaging platform designed to record RGB iris details in a video format by employing hyper-focal imaging and lateral white LED lighting resulting in a sharp, well exposed, and high DOF image of the eye. This overcomes some of the problems associated with capturing dark irides in visible light. A comparative study between single-focus and hyper-focal images is performed to demonstrate the positive effects of hyper-focal imaging in the context of visible spectrum iris biometrics.

\section{DATA ACQUISITION}

\section{A. Platform Design}

We designed a hyper-focal video recording platform using focus bracketing with 30 FPS 1080p HD video. A novel technique was developed to adjust the focus of the camera lens automatically in real time by programming an Okii FC1 Arduino-based follow focus controller (Okii Systems LLC, Apex, NC) to communicate with a Canon T2i dSLR's internal motorized focus mechanism through the camera's USB port. The Okii controlled Canon T2 $\mathrm{i}$ is mounted on a tripod setup while the subject uses a chin rest to face the lens and its attached lighting element (Figure 1). A single focus stack acquisition takes around 0.5 seconds at full HD resolution and a frame rate of $30 \mathrm{fps}$. Average distance from the camera to the subject is $1.5 \mathrm{ft}$. The camera is operated at $\mathrm{F}$ number $\mathrm{f} / 5.6$, an exposure time of $1 / 125 \mathrm{sec}$, and ISO of 800 .

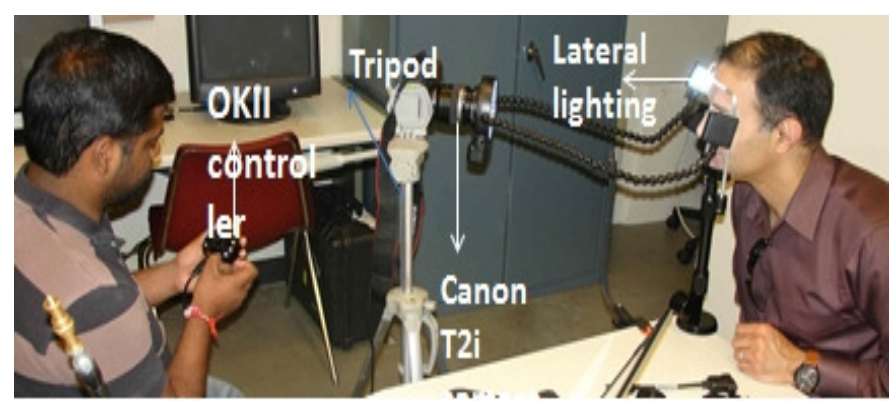

Fig. 1. The image acquisition setup that was adopted in this work. The images are acquired in a cooperative environment. Future work will include relaxing some of the acquisition constraints in order to obtain images from less cooperative subjects.

\section{B. Optics and illumination devices}

A modified Digi-Slave Flex Ring 6400 is used for illumination. This macro light is equipped with 64 oversized white LEDs, 32 LEDs in the ring (center light with $11 \mathrm{~cm}$ outer diameter and $7 \mathrm{~cm}$ inner diameter) and two flexible arms (modified to extend $50 \mathrm{~cm}$ long to the side of each eye) with 16 white LEDs on each pad (Figure 2). The light is used as in continuous mode to avoid photic startle and image artifacts. A polarizing filter film is applied to each side light pad for subject comfort. In this experiment we used only side lights for illumination and the center ring was turned off. Camera lens consisted of an EFS $50 \mathrm{~mm}, \mathrm{f} / 2.8$ macro lens and an Okii micro controller.

\section{Okii micro controller}

Okii micro controller is a programmable Aurdino-based USB follow-focus host device connected to the camera. It is powered by Atmel Atmega328P that runs at $8 \mathrm{MHz}$. Two focal points are saved as the start and stop points for focus stacking operations. Next, the speed of movement of the lens between these two focal points is determined by the number of steps required to move between these two points. The controller may be operated in small, medium and large focus step modes that correspond to the rate of motorized focus change introduced in the camera lens. Medium setting was used in this acquisition protocol. A button on the Okii controller is programmed to move the lens from a focal plane around subject's tip of the nose, which is defined as point A, to the vicinity of the ear, which is defined as point $\mathrm{B}$.

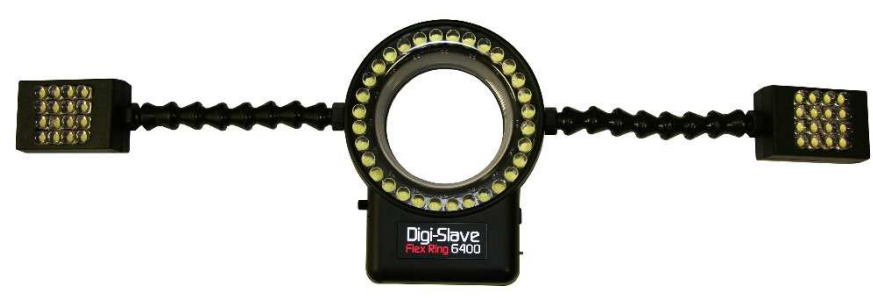

Fig. 2. Lateral LED illumination setup

\section{DATA COLLECTION PROTOCOL}

Data was collected from 46 volunteers based on IRB protocol 11-57e. Image stacks were collected over two sessions for each subject with a time gap of 45 minutes between sessions. Analysis was performed based on video recordings captured in session 1 and session 2. The data is acquired in a dark environment to avoid external artifacts. Lateral LED lighting pads were used to illuminate the iris as shown in Figure 1. It is hypothesized that this lighting configuration and location provides good textural information since the 3D iris structure casts more shadows at such incident angles compared to frontal illumination, thus accentuating the iridial textures. Furthermore, the indirect lighting angle alleviates subject discomfort despite the proximity of the pads to the iris. Subjects were asked to place their head on the chin rest while data collection was in progress. Note that this study was conducted under controlled conditions using cooperative users in order to establish the benefits of hyper-focal imaging. Future work will explore the use of a less controlled acquisition environment. Each recording included movements of focal point from tip of the nose (point A) to end of the ear (point B) labeled as round 1, and back (from B to A) labeled as round 2. The total recording time is one second $(0.5 \mathrm{sec}$ for each round). The acquired video at the rate of $30 \mathrm{fps}$ provides sufficient number of frames for hyper-focal processing in one complete pass. In each session, subjects were asked to look straight at the camera during the recordings. A sequence of frames was extracted from the captured 1-second clip [9]. 
From the extracted frames, we used only 8 frames per direction i.e. 8 in the forward direction (from $\mathrm{A}$ to $\mathrm{B}$ ) and 8 in the reverse direction (from $\mathrm{B}$ to $\mathrm{A}$ ). In our study we used two fused samples, one pertaining to the forward direction and one pertaining to the reverse direction. The analysis is performed using two fused samples from session 1 and two fused samples from session 2 for each subject.

\section{IMAGE FUSION USING HELICON FOCUS}

A sequence (focus stack) of 8 frames obtained at varying focal planes is fused to result in a sharp, high DOF hyper-focal image. The 8 consecutive frames are manually selected from the acquired video based on the prototype shown in Figure 3.

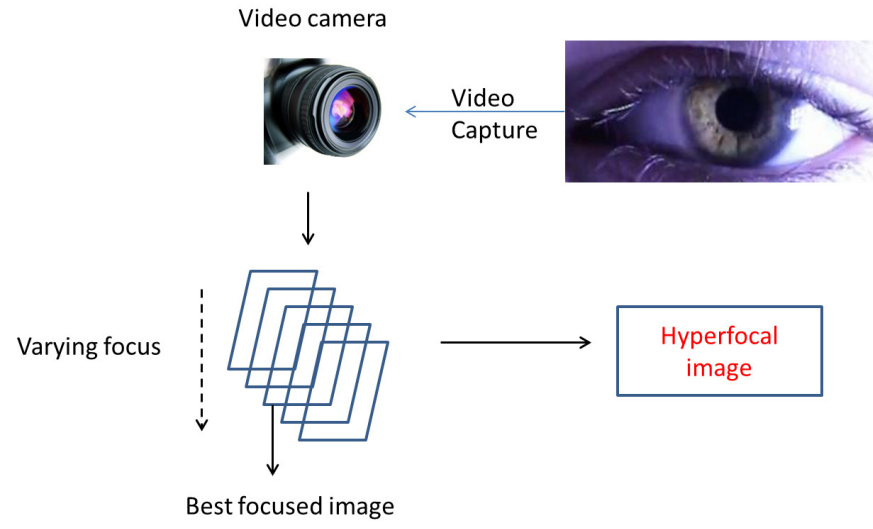

Fig. 3. Fusion prototype using the hyper-focal acquisition system

The Helicon focus software (Helicon Soft Ltd., Kharkov, Ukraine) [1] uses information from the sequence of images in the focus stack and produces a single hyper-focal image by fusing the sharper areas from each frame while adjusting for subtle movement and scaling aberrations across focal planes. Helicon focus software provides two options for focus stack fusion. Method A computes fusion weights for each pixel based on their contrast information, and all the corresponding pixel weights are averaged across the sequence of frames. Method B uses the sharpest pixels information and produces a depth map for stack fusion. This method has an advantage of overcoming halo effects across contrast edges. The focus measure at each point is determined by the distribution of pixels at a certain distance $\mathrm{R}$ from the point under consideration. Smoothing is another fusion parameter determining how smoothly the focus areas across stack captures are combined in the final hyper-focal image. This software registers the input images before applying the above mentioned methods. In our study we used Method $B$ with a radius of $R=45$ pixels and a default smoothing value of 4 . The exact method of registration and matching are proprietary.

Figure 4(b)(d)(f) shows examples of hyper-focal fusion using the Helicon focus software.

\section{IRIS SEGMENTATION AND MATCHING}

Ocular regions for left and right eyes were manually cropped from every acquired face image. The original face image had dimensions of $1080 \times 1920$ pixels and iris region in the cropped ocular images had an average of 82 pixels across its diameter. The final dataset has 184 iris images for each eye corresponding to 4 fused samples per subject for 46 subjects. These 4 fused samples are obtained over two sessions with 2 fused samples per session: one fused sample is generated from frames in the forward direction (round 1) and the other fused sample is generated from frames in the reverse direction (round 2).

The red channel image is used for iris matching as this is assumed to reveal maximum textural information due to its proximity to the NIR spectrum. The iris images are segmented by approximating the limbic boundary and pupillary boundary as circles and searching for the largest gradient values in circular Hough space. The upper and lower eye lids are approximated with straight lines. More details of the segmentation process can be found in [10].

Improperly segmented irides are visually determined and are manually rectified. This is to avoid issues related to incorrect segmentation that can affect the subsequent matching results. Pupillary boundary for darker irides is virtually indistinguishable from the surrounding iris region in RGB and poses a major problem for automatic segmentation. Segmented iris images are unwrapped to a pseudo-polar rectangular grid using Daugman's rubber sheet model [4]. The gray scale rectangular normalized image is converted to an IrisCode [4] using Masek's encoding method [10] that uses log-Gabor filters in the Fourier domain and further quantization to convert the filter response to a binary code known as IrisCode. Normalized Hamming distance is used to measure the dissimilarity between two IrisCodes. Thus, a lower match score indicates a better match. Several match scores are generated by computing the Hamming distance between one IrisCode and horizontally shifted versions of the other and the least score is selected as the final match score. This accounts for rotational inconsistencies between the two iris images.

Figure 5 shows the result of segmentation and normalization on a sample iris image.

\section{Focus MEASURE}

A focus metric is used to compare the quality of the acquired sequence of frames with their corresponding hyper-focal fused image. We used a simple wavelet based focus metric [8] in this study. It is noted that focus index of an image is highly correlated with sum of its wavelet coefficients, where a higher sum indicates better focus. Wavelet coefficients are generated based on equation below, where $\mathrm{I}(\mathrm{X}, \mathrm{Y})$ is the input image and $\psi$ is the basis function:

$$
\begin{gathered}
W_{\psi}^{i}(j, m, n)=\frac{1}{M N} \sum_{x=0}^{M-1} \sum_{y=0}^{N-1} I(x, y) \psi_{j, m, n}(x, y) \\
i=\{H, V, D\} \\
I_{f}=\operatorname{Mean}\left(\sum W_{\psi}^{i}(j, m, n)\right), i=\{H, V, D\}
\end{gathered}
$$



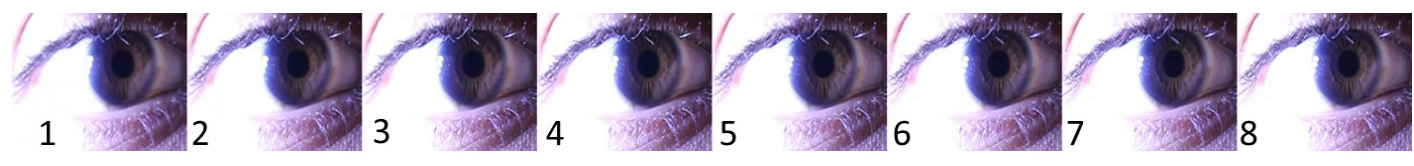

(a) - Sequence 1
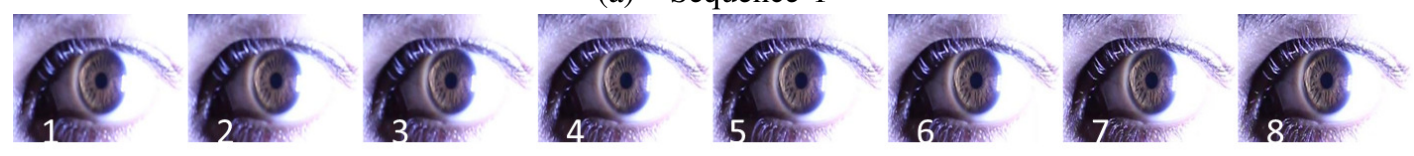

(c) - Sequence 2
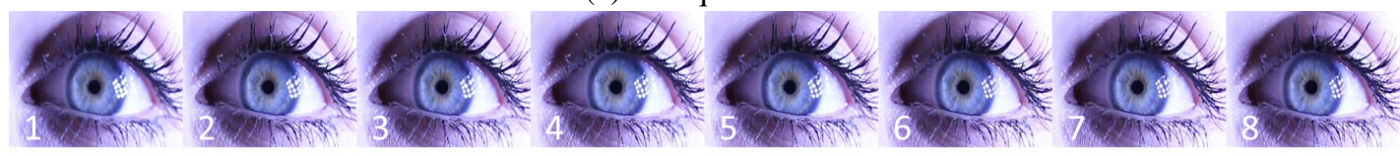

(e) - Sequence 3

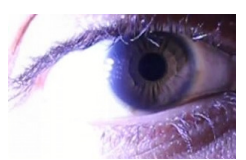

(b)

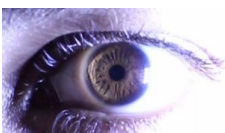

(d)

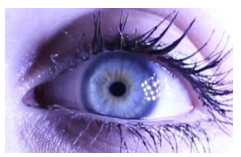

(f)

Fig. 4. Examples for individual frame sequences and corresponding hyper-focal fused images. Here, (a), (c) and (e) represent 3 sets of input frames. (b), (d) and (f) are the corresponding fused frames.

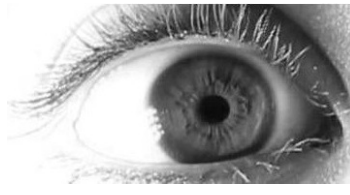

(a)

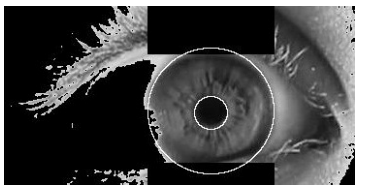

(b)

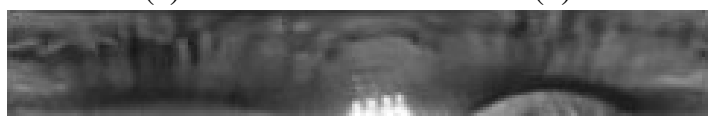

(c)

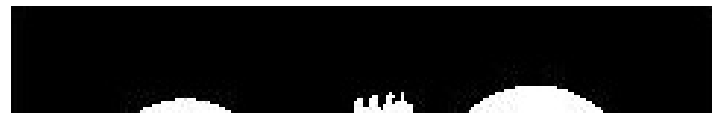

(d)

Fig. 5. (a) Original image (b) Segmentation output (c) Normalized image and (d) corresponding mask image

Wavelet decomposition of image in the horizontal $(\mathrm{H})$, Vertical (V) and diagonal (D) directions is performed using Daubechies mother wavelet of order one. $I_{f}$ is the mean of the wavelet coefficients in all the directions resulting in our focus metric. Figure 6 shows the focus values for individual frames in the video sequence shown in Figure 4 (focus stack) and the corresponding value for fused hyper-focal image. It is evident that hyper-focal fusion yields a higher focus measure for fused image compared to the input frames.

\section{EXPERIMENTS}

We compared the performance of single focus and hyperfocal images independently on the left and right eyes. In this analysis we chose the middle frame (4th frame) from the 8frame sequence and generated iris match scores on the dataset. Choosing the fused hyper-focal image for matching resulted in a decreased EER compared to matching using only the middle frame. Figure 7 shows the plotted ROC curves and Table I shows the corresponding EER values for middle frame and

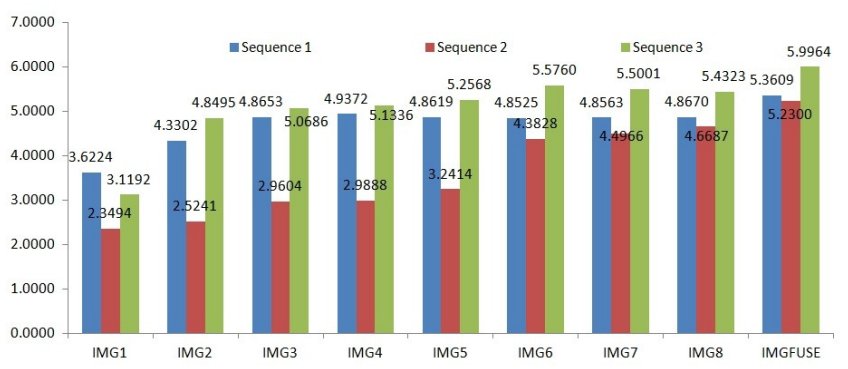

Fig. 6. Focus measure for individual frames against the fused hyper-focal image

fused hyper-focal image matching.

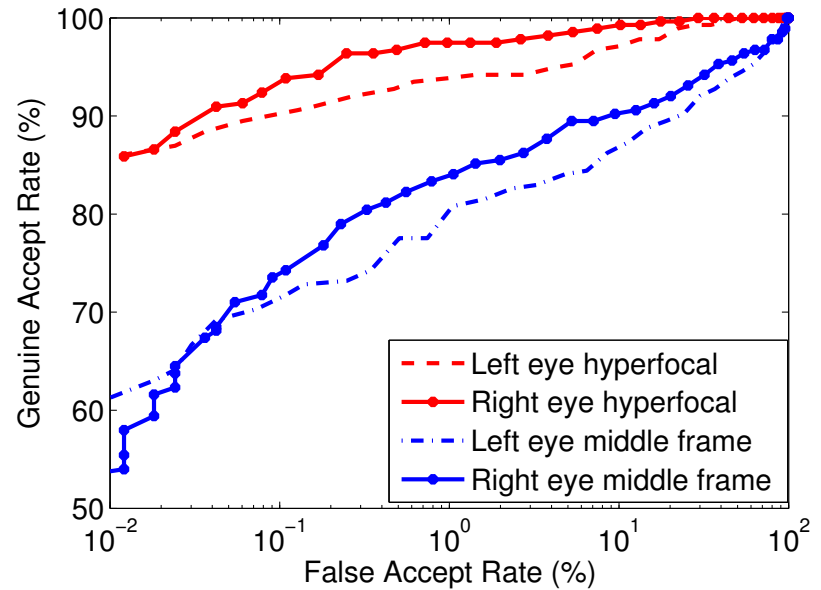

Fig. 7. ROC curves for left and right eye of Hyper-focal images and middle frame from the stack of variable focus images

To illustrate the effect of focus of the captured image on the iris match scores, box plots are shown in Figure 8.

For each subject, 8 frames are available in the first sequence 


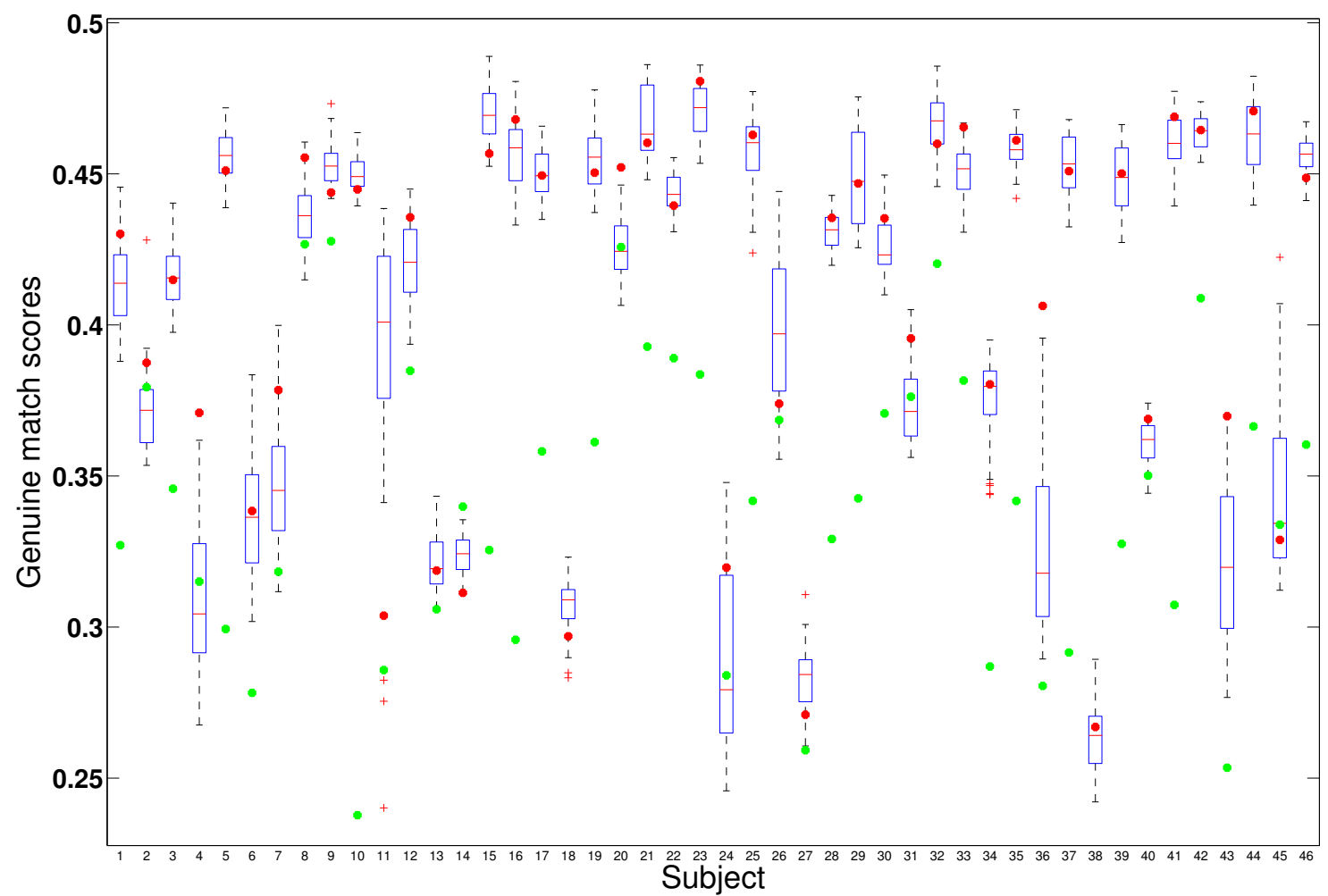

Fig. 8. Box plots of genuine match scores for all the 46 subjects for session 1 against session 2

TABLE I

ROC ANALYSIS FOR HYPER-FOCAL IMAGES AND MIDDLE FRAMES FROM SEQUENCE OF 8 FRAMES USED FOR HYPER-FOCAL FUSION

\begin{tabular}{|c|c|c|}
\hline & $\begin{array}{c}\text { Hyper-focal } \\
\text { images }\end{array}$ & $\begin{array}{c}\text { Fixed focus } \\
\text { images }\end{array}$ \\
\hline & EER & EER \\
\hline Left Eye & 0.04997 & 0.1245 \\
\hline Right Eye & 0.021588 & 0.096347 \\
\hline
\end{tabular}

each of Session 1 and Session 2. Genuine match scores are generated by matching all the 8 frames from first sequence of Session 1 against all the 8 frames from the first sequence of Session 2. Figure 8 shows the box plots of these genuine scores along with the match score (red dot) for matching the best focused frames in both the sessions and corresponding match score (green dot) for fused hyper-focal images. It is clearly evident as shown in Figure 8, that the genuine match score for the fused hyper-focal image is much lower than that of the middle frame, there by suggesting a better match.

Wilcoxon signed rank test [18] is used to demonstrate the statistical significance of the improvement in genuine match score when using hyper-focal image compared to single focus images (middle frame). Table II shows the z-values and the corresponding p-values for different scenarios. S1(S2) stands for Session 1(Session 2) and R1(R2) stands for the first(second) sequence.

TABLE II

STATISTICAL SIGNIFICANCE TABLE FOR HYPER-FOCAL IMAGE VS. BEST FOCUSED IMAGE (WILCOXON TEST RESULTS)

\begin{tabular}{|c|c|c|c|c|}
\hline & $\begin{array}{c}\text { S1R1 } \\
\text { vs } \\
\text { S2R1 }\end{array}$ & $\begin{array}{c}\text { S1R1 } \\
\text { vs } \\
\text { S2R2 }\end{array}$ & $\begin{array}{c}\text { S1R2 } \\
\text { vs } \\
\text { S2R1 }\end{array}$ & $\begin{array}{c}\text { S1R2 } \\
\text { vs } \\
\text { S2R2 }\end{array}$ \\
\hline z-statistic & 4.4166 & 3.2694 & 4.4603 & 4.6351 \\
\hline p-value & $<0.0001$ & 0.0005 & $<0.0001$ & $<0.0001$ \\
\hline
\end{tabular}

Values in Table II reject the null hypothesis that the genuine match score of hyper-focal image is greater than genuine match score for middle frame of the 8 selected frames. Another scenario is considered where hyper-focal match score is compared against the minimum possible match score for a fixed focus frame. Assuming the basic assumptions for t-test to be true, Table III shows the significance of hyper-focal images in iris recognition.

Table III shows that genuine score for fused hyper-focal image is lower(better) than the best possible genuine score for fixed focus image scenario with a high statistical significance. 
TABLE III

STATISTICAL SIGNIFICANCE TABLE FOR HYPER-FOCAL IMAGE VS. BEST MATCH SCORE USING A SINGLE FIXED FOCUS IMAGE

\begin{tabular}{|c|c|c|c|c|}
\hline & $\begin{array}{c}\text { S1R1 } \\
\text { vs } \\
\text { S2R1 }\end{array}$ & $\begin{array}{c}\text { S1R1 } \\
\text { vs } \\
\text { S2R2 }\end{array}$ & $\begin{array}{c}\text { S1R2 } \\
\text { vs } \\
\text { S2R1 }\end{array}$ & $\begin{array}{c}\text { S1R2 } \\
\text { vs } \\
\text { S2R2 }\end{array}$ \\
\hline t-statistic & 2.3714 & 2.4330 & 2.7123 & 2.3448 \\
\hline p-value & 0.0110 & 0.0095 & 0.0047 & 0.0117 \\
\hline
\end{tabular}

\section{DISCUSSION}

A sequence of 8 images captured in a fraction of a second was used for fusion in our study. However, this number depends on the native frame rate of the camera, 3D depth of an eye, and also speed of the motorized lens moving mechanism. Higher native speed of the camera would ultimately reduce the chances of observing motion blur and occlusions in image acquisition. We used 1080p HD video recordings with $30 \mathrm{fps}$ instead of the regular burst mode full frame image captures and used the lens' internal focus motors for smoother lens movements. This obviates the use of moving rails and external motor-operated lens moving mechanisms. These traditional mechanisms when operated at higher speeds might induce vibration resulting in motion artifacts. Another instrumental factor in our design is the use of side lighting making RGB iris captures a possibility. Our statistical analysis and ROC analysis clearly show that hyper-focal imaging performs better than regular imaging. Thus, hyper-focal imaging technique in conjunction with existing commercial technologies would help in improving iris recognition rates.

\section{CONClusion And Future wOrk}

In this paper we introduced a novel image acquisition platform using hyper-focal imaging technique and side lighting to acquire high quality images of iris in the visible spectrum with greater DOF. Statistical analysis confirm, with high confidence, that hyper-focal imaging systems produce better results compared to single focus systems. In future we would like to implement this technique to see the effect of imaging for on iris-on-the-move systems. Using these high-resolution RGB iris images a study on pigmentation assessment using iris color information will be performed in the future.

\section{AcKnowledgments}

Research was sponsored by the Leonard Wood Institute in cooperation with the U.S. Army Research Laboratory and was accomplished under Cooperative Agreement Number W911NF-07-2-0062. The views and conclusions contained in this document are those of the authors and should not be interpreted as representing the official policies, either expressed or implied, of the Leonard Wood Institute, the Army Research Laboratory or the U.S. Government. The U.S. Government is authorized to reproduce and distribute reprints for Government purposes notwithstanding any copyright notation heron. Authors thank Bret Lesan, CMfgE, Senior Research and Design Engineer, UMKC for help with mechanical design of the acquisition system and Plamen Doynov for assisting with preparation of the initial draft.

\section{REFERENCES}

[1] "Helicon Soft". Helicon soft ltd. http://www.heliconsoft.com/.

[2] K.W. Bowyer, K. Hollingsworth, and P.J. Flynn. Image understanding for iris biometrics: A survey. Computer Vision and Image Understanding, 110(2):281-307, 2008.

[3] C. Boyce, A. Ross, M. Monaco, L. Hornak, and X. Li. Multispectral iris analysis: A preliminary study. In Computer Vision and Pattern Recognition Workshop, page 51. IEEE, 2006.

[4] J. Daugman. How iris recognition works. IEEE Transactions on Circuits and Systems for Video Technology, 14(1):21-30, 2004.

[5] Y. He, J. Cui, T. Tan, and Y. Wang. Key techniques and methods for imaging iris in focus. In 18th International Conference on Pattern Recognition, volume 4, pages 557-561. IEEE, 2006.

[6] N.D. Kalka, J. Zuo, N.A. Schmid, and B. Cukic. Estimating and fusing quality factors for iris biometric images. IEEE Transactions on Systems, Man and Cybernetics, Part A: Systems and Humans, 40(3):509-524, 2010.

[7] E. Krichen, M.A. Mellakh, S. Garcia-Salicetti, and B. Dorizzi. Iris identification using wavelet packets. In IEEE Proceedings of the 17th International Conference on Pattern Recognition, volume 4, pages 335338, 2004.

[8] J.H. Lee, K.S. Kim, B.D. Nam, J.C. Lee, Y.M. Kwon, and H.G. Kim Implementation of a passive automatic focusing algorithm for digital still camera. IEEE Transactions on Consumer Electronics, 41(3):449-454, 1995.

[9] DVDVideoSoft Limited. ://www.dvdvideosoft.com/products/dvd/ free-video-to-jpgconverter.htm.

[10] Libor Masek and Peter Kovesi. Matlab source code for a biometric identification system based on iris patterns, 2003.

[11] Ramkumar Narayanswamy and Paulo E.X. Silveira. Iris recognition at a distance with expanded imaging volume. Biometric Technology for Human Identification - II, 5779:41-50, 2005.

[12] K.R. Park and J. Kim. A real-time focusing algorithm for iris recognition camera. IEEE Transactions on Systems, Man, and Cybernetics, Part C: Applications and Reviews, , 35(3):441-444, 2005.

[13] R. Plemmons, M. Horvath, E. Leonhardt, P. Pauca, S. Prasad, S. Robinson, H. Setty, T. Torgersen, J. van der Gracht, E. Dowski, et al. Computational imaging systems for iris recognition. In Proc. SPIE, volume 5559, pages 346-357, 2004.

[14] H. Proença and L. Alexandre. UBIRIS: A noisy iris image database. ICIAP, pages 970-977, 2005.

[15] P. Radu, K. Sirlantzis, WGJ Howells, S. Hoque, and F. Deravi. Information fusion for unconstrained iris recognition. International Journal of Hybrid Information Technology, 4(4):1-12, 2011.

[16] A. Ross. Iris recognition: The path forward. Computer, 43(2):30-35, 2010.

[17] K.N. Smith, V.P. Pauca, A. Ross, T. Torgersen, and M.C. King. Extended evaluation of simulated wavefront coding technology in iris recognition. In International Conference on Biometrics: Theory, Applications, and Systems, pages 1-7. IEEE, 2007.

[18] F. Wilcoxon. Individual comparisons by ranking methods. Biometrics Bulletin, 1(6):80-83, 1945. 